\title{
Involvement of a Class III Peroxidase and the Mitochondrial Protein TSPO in Oxidative Burst Upon Treatment of Moss Plants with a Fungal Elicitor
}

\author{
Mikko T. Lehtonen, ${ }^{1}$ Motomu Akita, ${ }^{1,2}$ Wolfgang Frank, ${ }^{3}$ Ralf Reski, ${ }^{3,4,5}$ and Jari P. T. Valkonen ${ }^{1}$ \\ ${ }^{1}$ Plant Pathology Laboratory, Department of Agricultural Sciences, PO Box 27, Fl-00014 University of Helsinki, Finland; \\ 2Department of Biotechnological Sciences, Kinki University, Kinokawa, Wakayama, 649-6493, Japan; ${ }^{3}$ Plant Biotechnology, \\ Faculty of Biology, University of Freiburg, Schaenzlestr. 1, ${ }^{4} \mathrm{BIOSS}$ Centre for Biological Signalling Studies, University of \\ Freiburg, Hebelstr. 25, and ${ }^{5}$ FRIAS Freiburg Institute for Advanced Studies, Albertstr. 19, D-79104 Freiburg, Germany
}

Submitted 11 October 2011. Accepted 11 November 2011.

Production of apoplastic reactive oxygen species (ROS), or oxidative burst, is among the first responses of plants upon recognition of microorganisms. It requires peroxidase or NADPH oxidase (NOX) activity and factors maintaining cellular redox homeostasis. Here, PpTSPO1 involved in mitochondrial tetrapyrrole transport and abiotic (salt) stress tolerance was tested for its role in biotic stress in Physcomitrella patens, a nonvascular plant (moss). The fungal elicitor chitin caused an immediate oxidative burst in wild-type $P$. patens but not in the previously described $\Delta$ Prx34 mutants lacking the chitin-responsive secreted class III peroxidase (Prx34). Oxidative burst in $P$. patens was associated with induction of the oxidative stressrelated genes $A O X, L O X 7$, and $N O X$, and also PpTSPO1. The available $\triangle P p T S P O 1$ knockout mutants overexpressed $A O X$ and $L O X 7$ constitutively, produced 2.6 -fold more ROS than wild-type $P$. patens, and exhibited increased sensitivity to a fungal necrotrophic pathogen and a saprophyte. These results indicate that Prx34, which is pivotal for antifungal resistance, catalyzes $\mathrm{ROS}$ production in $P$. patens, while PpTSPO1 controls redox homeostasis. The capacity of TSPO to bind harmful free heme and porphyrins and scavenge them through autophagy, as shown in Arabidopsis under abiotic stress, seems important to maintenance of the homeostasis required for efficient pathogen defense.

Plants sense their environment and react to abiotic and biotic stimuli with a wide range of physiological and biochemical responses. Apoplastic reactive oxygen species (ROS) are among the first molecules produced upon recognition of microorganisms by the plant (Nanda et al. 2010; Torres 2010). Rapid ROS generation, known as the oxidative burst, is observed commonly upon pathogen attack (Apel and Hirt 2004; Wojtaszek 1997) and results in fast systemic activation of pathogen defense in the plant (Miller et al. 2009). In addition to their signaling effect, ROS can be toxic to the invading microbes (Angelova et al. 2005), mediate production of phytoalexins and other compounds that limit the growth of pathogens, and be positive or negative regulators of the hypersensitive response (HR) associ-

Corresponding author: J. P. T. Valkonen; E-mail: jari.valkonen@ helsinki.fi; Telephone: +358-9-19158387

* The $\boldsymbol{e}$-Xtra logo stands for "electronic extra" and indicates that two supplementary figures are published online. ated with cell death (Torres 2010). Although HR may restrict the pathogen to the initial infection site, necrotrophic fungal pathogens can take advantage of the HR-associated cell death and use it as a means to colonize the host plant (Govrin and Levine 2000).

The origins of extracellular ROS seem to vary depending on the plant-pathogen combination and have been attributed to, for example, an apoplastic peroxidase (Bindschedler et al. 2006) and a NADPH oxidase localized in the plasma membrane (Torres et al. 2002). Peroxidases utilize hydrogen peroxide $\left(\mathrm{H}_{2} \mathrm{O}_{2}\right)$ for oxidative power in the peroxidative cycle but they are also capable of producing highly reactive free radicals such as superoxide in the hydroxylic cycle for defense purposes (Kawano 2003).

The signaling pathways involving the peroxidase or NADPH oxidase-catalyzed superoxide production in vascular plants include common factors (Fujita et al. 2006; Walley et al. 2007). One of them is the heme co-factor that is required by peroxidases and NADPH oxidases for electron transfer in ROS production (Keller et al. 1998; Schuller et al. 1996). Heme is produced via the tetrapyrrole biosynthesis pathway in plastids (Mochizuki et al. 2010). Peripheral-type benzodiazepine receptor and the bacterial tryptophane-rich sensory protein are involved in the transport of intermediates of the tetrapyrrole biosynthesis pathway, and a gene encoding a homologous protein (PpTSPO1) has been described in a moss (Physcomitrella patens (Hedw.) B. S. G., family Funariaceae), a nonvascular plant (Frank et al. 2007). The PpTSPO1 protein is localized to mitochondria, induced by drought and abscissic acid, and essential for adaptation of the moss to salt stress. The PpTSPO1 knockout mutants are impaired in their mitochondrial protoporphyrin IX uptake and produce elevated levels of intracellular ROS, implying a role of PpTSPO1 in redox homeostasis (Frank et al. 2007). Recently, the homolog of PpTSPO1 has been characterized in Arabidopsis thaliana, in which it contributes to salt stress tolerance (Balsemão-Pires et al. 2011) as in P. patens, and is involved in scavenging unbound heme and porphyrins (Vanhee et al. 2011).

Mosses produce ROS, including $\mathrm{H}_{2} \mathrm{O}_{2}$, under abiotic stress (Frank et al. 2007; Mayaba et al. 2002; Proctor et al. 2007) but little is known about production of ROS during biotic stresses in mosses. P. patens encodes a class III peroxidase (Prx34) that is released immediately to the extracellular space and culture medium upon treatment of the moss with a chitosan preparation (CHN) (Lehtonen et al. 2009). The CHN preparation contains chitin, a fungal cell wall component that elicits pathogen 
defense in plants, and which is deacetylated to $90 \%$ in order to make it soluble in water (Knaul et al. 1999; Shibuya and Minami 2001). In the first and, thus far, only study that has utilized knockout mutants of $P$. patens to address questions related to pathogen defense and biotic stresses (Lehtonen et al. 2009), the two copies of the Prx34 gene were deleted utilizing the highly efficient homologous recombination in $P$. patens and the dominant haploid lifestyle to reveal the phenotypes (Cove 2005; Strepp et al. 1998). Results showed that the 11 independent $\triangle$ Prx34 mutants tested had lost resistance to pathogenic and saprotrophic fungi isolated from mosses. However, i) whether or not apoplastic oxidative burst occurs in mosses as a defense response to infection (biotic stress) and ii) whether Prx34 is involved is not known. Similarly, iii) whether the functions of the mitochondrial protein TSPO are important in biotic stress has not been reported. The aim of this study was to answer the three aforementioned question using $P$. patens as the model system.
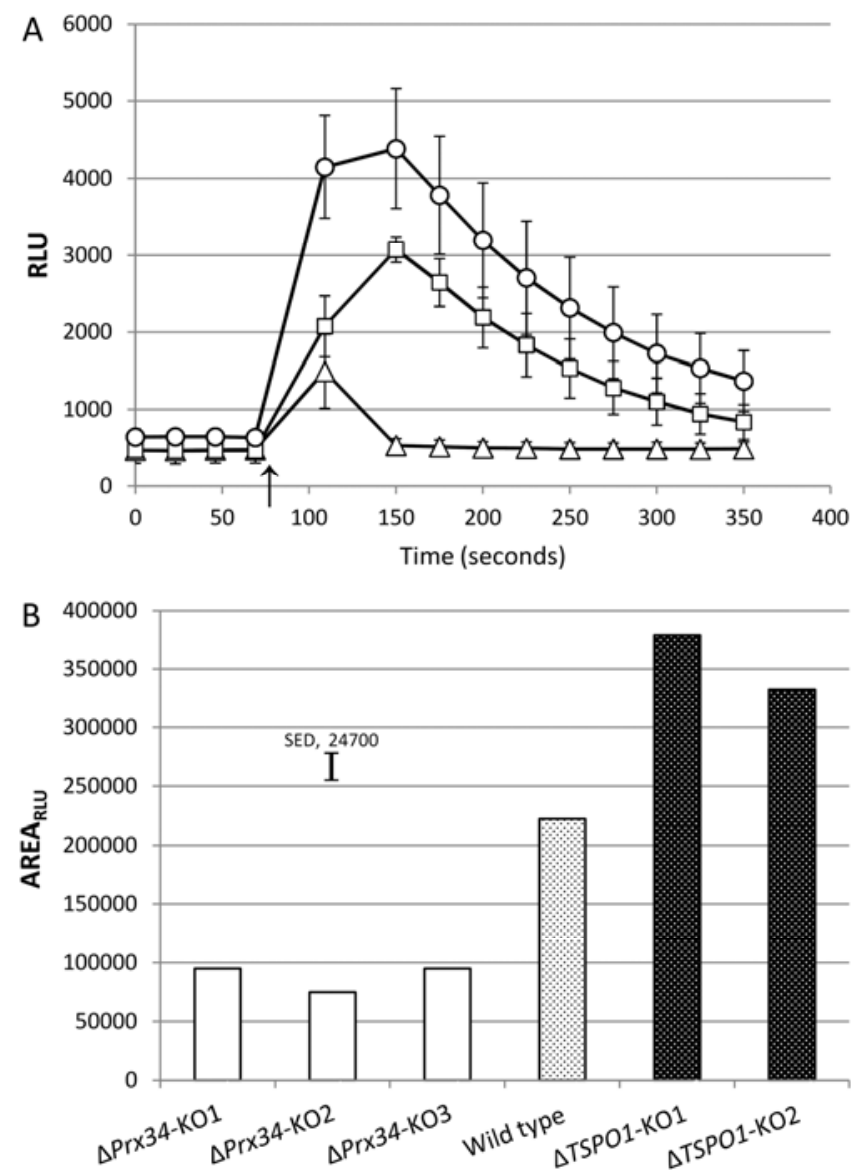

Fig. 1. Production of superoxide $\left(\mathrm{O}_{2}{ }^{-}\right)$in Physcomitrella patens upon elicitation with a chitosan preparation $(\mathrm{CHN}) . \mathbf{A}, \mathrm{O}_{2}{ }^{-}$measured as relative light units (RLU) based on chemiluminescence of the luciferin analog 2-methyl-6(p-methoxyphenyl)-3,7-dihydroimidazo(1,2- $\alpha$ )pyrazin-3-one at different time points following addition of $\mathrm{CHN}$ to the liquid culture medium in four independent experiments. Arrow indicates the time point of $\mathrm{CHN}$ addition. Symbols: $\square$ = wild-type (WT) P. patens; $O=\triangle P p T S P O 1$, a knockout mutant that is lacking the TSPO1 gene and exhibits sensitivity to salt stress (Frank et al. 2007); $\triangle=\Delta \operatorname{Prx} 34$, a knockout mutant lacking the CHN-responsive class III peroxidase gene and rendered susceptible to fungal infection (Lehtonen et al. 2009). Error bars indicate standard deviation $(n=4)$. B, Mean cumulative $\mathrm{O}_{2}{ }^{-}$production in three independent experiments at $3 \mathrm{~min}$ after addition of $\mathrm{CHN}$ to the culture medium. Three independent $\triangle \operatorname{Prx} 34$ knockout mutants, WT $P$. patens, and two independent $\triangle P p T S P O 1$ knockout lines were tested. Differences between the $\triangle P r x 34$ and $\triangle P P T S P O 1$ knockout lines and WT $P$. patens are statistically significant $(P<0.01)$. The error bar indicates standard error of difference (SED; $\mathrm{df}=220$ ).

\section{RESULTS}

Superoxide production induced by $\mathrm{CHN}$ in $P$. patens.

There is little information available from nonvascular plants as to whether or not they respond to pathogens or pathogen elicitors with oxidative burst. It was tested in this study by application of CHN, a water-soluble hydrolysate of the fungal cell wall component chitin, to the liquid culture of $P$. patens. An immediate burst of ROS in the medium was observed (Fig. 1A). Superoxide production also was detected in the axenic cultures of Sphagnum capillifolium (Ehr.) Hedw. (Supplementary Fig. S1), an unrelated moss species (family Sphagnaceae), upon treatment with CHN. These results indicate that oxidative burst is a general response of bryophytes to pathogens or pathogen elicitors.

Production of superoxide increased in a dose-dependent manner and reached the maximum level with application of CHN in the culture medium at the final concentration of $1 \mathrm{mg}$ $\mathrm{ml}^{-1}$ (Supplementary Fig. S2A). However, the oxidative burst was not observed in the protoplasts of $P$. patens treated with $\mathrm{CHN}$, suggesting that the secreted peroxidase is deposited to cell walls that are removed in the process of protoplast preparation.

Dependence of the ROS generation on Prx34 was tested by including the previously described $\Delta \operatorname{Pr} x 34$ knockout mutants, in which the gene for a CHN-induced, excreted peroxidase Prx34 has been knocked out (Lehtonen et al. 2009). There was no oxidative burst observed with $\Delta \operatorname{Pr} x 34$ knockout mutants in four independent experiments (Fig. 1A), indicating that Prx34 is an essential catalyst of ROS production.

The total amount of superoxide that accumulated during the first $3 \mathrm{~min}$ postapplication of $\mathrm{CHN}$ in three independent experiments indicated that superoxide production in the two independent, previously described $P$ PTSPOI knockout mutant lines ( $\triangle P$ PTSPOI) (Frank et al. 2007) was 1.6-fold higher than that in the wild-type (WT) P. patens (Fig. 1B). On the other hand, superoxide production in the three independent $\Delta \operatorname{Pr} x 34$ mutants tested was only $40 \%$ of that in WT P. patens (Fig. 1B) and was not affected by CHN treatment (Fig. 1A). The consistent response among the independent knockout mutant lines in repeated experiments and the statistically significant differences compared with WT P. patens (Fig. 1) indicated that the increased or decreased superoxide production resulted from the deletion of PpTSPOI and Prx34 genes, respectively.

Superoxide production correlates with peroxidase activity.

Because the results on the $\Delta \operatorname{Prx} 34$ knockout mutants indicated that the oxidative burst upon treatment with $\mathrm{CHN}$ requires Prx34 (Fig. 1), we tested whether the higher ROS production in $\triangle P$ PSPOI also was associated with higher peroxidase activity. Indeed, peroxidase activity in the culture medium of the $\triangle P p T S P O 1$ mutants was approximately 2.6 -fold higher than that of WT P. patens following treatment with CHN. The difference was statistically significant in the three independent experiments carried out (Fig. 2). These results suggested that the $\triangle P$ PTSPO1 mutants are not able to control ROS production, compared with WT $P$. patens.

\section{Elevated transcript levels of stress-associated genes in $\triangle P p T S P O 1$ mutants.}

Mosses (bryophytes) consist of approximately 10,000 species (Cuming 2009) but, still, only a very few studies have examined the molecular defense responses in P. patens following pathogen infection (Lawton and Saidasan 2009; Oliver et al. 2009; Ponce de León et al. 2007) or treatment with defense elicitors, such as salicylic acid (Andersson et al. 2005) or CHN (Lehtonen et al. 2009). 
Discovery of the extracellular ROS production in response to $\mathrm{CHN}$ treatment in this study was followed by studying the possible induction of ROS-responsive and defense-signalingrelated genes (Torres 2010), namely those encoding alternative oxidase $(P p A O X)$, NADPH-oxidase $(P p N O X)$, and lipoxygenase (PpLOX7). PpNOX and PpAOX are probable orthologs. These genes were identified in the genome sequence of $P$. patens (Rensing et al. 2008) and specific primers designed for quantitative reverse-transcription real-time polymerase chain reaction (PCR) analysis of gene expression. In the three experiments, the transcript levels of the three oxidative stress-associated genes were enhanced in WT P. patens treated with CHN (Fig. 3).

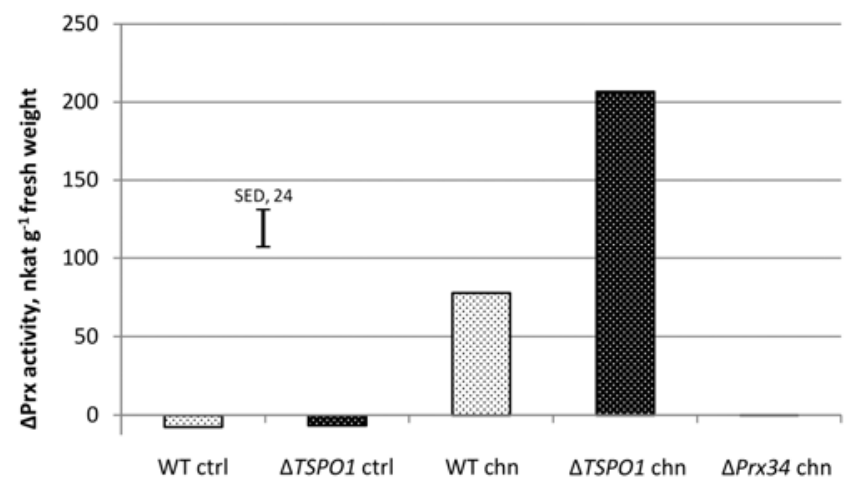

Fig. 2. Chitosan preparation (CHN)-induced peroxidase activity in moss lines. Bars indicate the change in peroxidase activity (activity $\mathrm{T}_{15}-\mathrm{T}_{0}$ ) measured in the liquid culture medium 15 min following addition of $\mathrm{CHN}$ (chn) or water (ctrl) to liquid cultures of wild-type (WT) Physcomitrella patens, $\triangle$ PpTSPO1 mutant, and $\triangle$ Prx34 mutant in three independent experiments. Differences between the three types of moss lines are statistically significant $(P<0.01)$. The error bar indicates standard error of difference $(\mathrm{SED} ; \mathrm{df}=27)$.

\section{A}

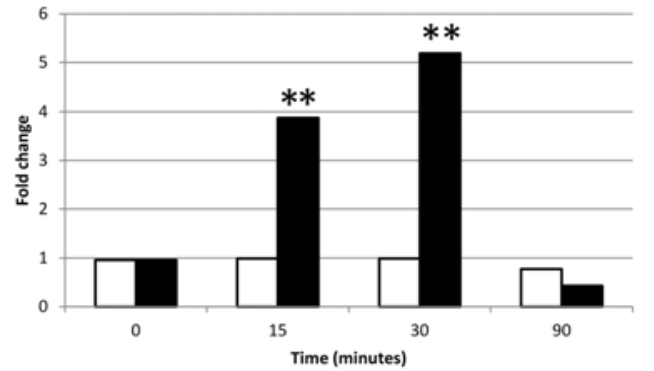

C

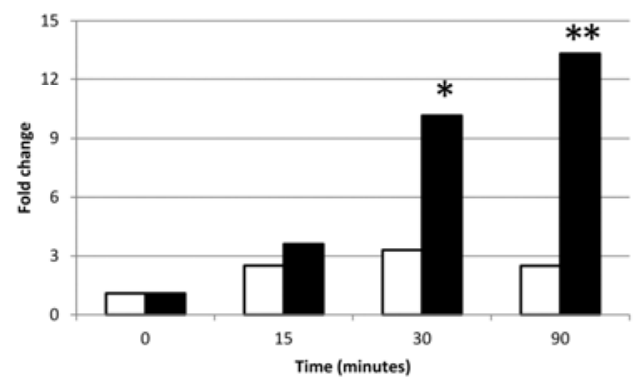

$P p A O X, P p N O X$, and $P p L O X 7$ transcript levels were slightly increased by $15 \mathrm{~min}$ and significantly increased by 30 and 90 min posttreatment with $\mathrm{CHN}$ (Fig. 3B to D). However, transcript levels of PpTSPO1 were elevated significantly as soon as 15 min posttreatment with $\mathrm{CHN}$ but returned to the basal level by $90 \mathrm{~min}$ after the $\mathrm{CHN}$ treatment (Fig. 3A).

Because the $\triangle P$ PTSPO1 mutants showed elevated ROS levels upon CHN treatment, we also analyzed transcript levels of the oxidative stress-related genes in these mutant lines. Data indicated that the transcript levels of PpAOX and PpLOX7 were constitutively overexpressed in the non-CHN-treated $\triangle P$ PTSO1 mutant compared with WT P. patens (Fig. 4). In contrast, expression levels of PpNOX and Prx34 in the $\triangle P$ PSPO1 mutants were slightly elevated but not significantly different from WT P. patens (Fig. 4).

\section{Resistance to fungi is reduced in $\triangle P$ PTSPO1.}

The higher constitutive expression of defense-related genes in the salt-sensitive $\triangle P$ PTSPO1 mutant was anticipated to also reflect higher levels of resistance to pathogens, which was tested by inoculation of the plants of $\triangle P$ PTSPOI and WT $P$. patens with two fungi representing a saprophytic minor pathogen (Irpex sp.) and a severe pathogen (Fusarium avenaceum), both isolated from moss (Akita et al. 2011; Lehtonen et al. 2009).

Fungal inoculum was placed next to the moss colony and hyphae were allowed to grow in contact with the moss protonema. Differences in the response between $\triangle P$ PTSPO1 and WT $P$. patens could be observed with a microscope as soon as the initial contact between fungal hyphae and the moss protonema (filamentous) tissue occurred (Fig. 5A and C). In $\triangle P$ PSPO1, discoloration of the transverse cell walls was pronounced at the contact points with $F$. avenaceum (Fig. 5D), whereas no discoloration was observed in WT P. patens (Fig. 5B). Later, at 12 days postinoculation, discoloration and death

B

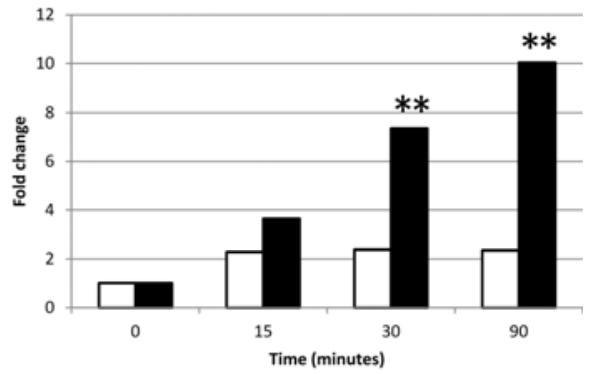

$\mathrm{D}$

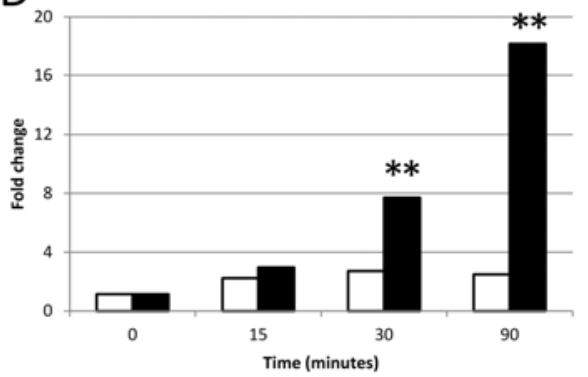

Fig. 3. Induction of TSPO1 and three reactive oxygen species (ROS)-responsive and defense signaling genes in Physcomitrella patens following treatment with a chitosan preparation (CHN). Transcript levels of the genes A, PpTSPO1; B, PpAOX; C, PpNOX; and D, PpLOX7 were determined by quantitative reverse-transcription real-time PCR (qRT-PCR) in WT P. patens treated with CHN (black bars) or water (control, white bars) at various times posttreatment. Bars indicate the mean fold changes in gene expression, compared with the expression level just before treatment (time $=0$ min), in three independent experiments. Asterisks indicate that the mean is significantly different from the non-treated control $(P<0.05 * ; P<0.01 * *)$. (ANOVA, $\mathrm{F}_{6,14}=21.1$ in $\mathrm{A}, 54.0$ in $\mathrm{B}, 41.4$ in $\mathrm{C}$, and 109.1 in $\mathrm{D} ; \alpha=0.001)$. Note that the fold change scale differs between charts. 
of protonema and also shoots were observed in the two $\triangle$ PpTSPOI lines tested (Fig. 6E, F, H, and I). Symptoms were more severe than in WT $P$. patens (Fig. 6B and C). These differences between the $\triangle P p T S P O 1$ lines and WT $P$. patens were consistent, no matter whether the Irpex sp. (Fig. 6B, E, and H) or F. avenaceum (Fig. 6C, F, and I) was used. Furthermore, the growth of mycelia of both fungal species was more abundant on the $\triangle$ PpTSPO1 lines than on WT P. patens (Fig. 6). These differences were found consistently in the four independent experiments carried out. Taken together, unexpectedly, $\triangle P$ PSPO1 was less resistant to both fungi compared with WT P. patens.
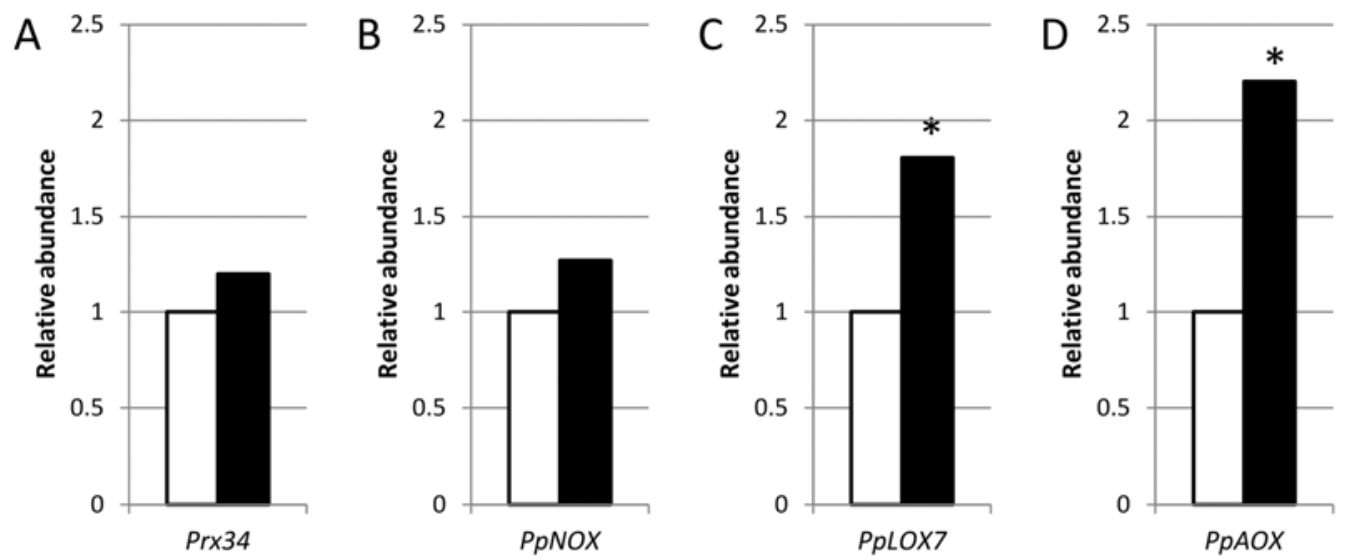

Fig. 4. Expression of stress-associated genes in wild-type (WT) Physcomitrella patens (white bar) as compared with expression in the $\triangle P p T S P O 1$ mutant lines (black bars) under normal growth conditions. Transcript levels were determined using quantitative reverse-transcription polymerase chain reaction and data normalized by comparison to EF1 $\alpha$ and $L 21$ transcripts. Bars indicate the mean of three independent experiments. Asterisks $(*)$ indicate that the mean of $\triangle P$ PTSPO1 is significantly different from WT P. patens $(P<0.01)$. Analysis of variance: $\mathrm{F}_{1,4}$ equals $\mathbf{A}, 2.11, \mathbf{B}, 3.29, \mathbf{C}, 25.1$, and $\mathbf{D}, 83.3$.

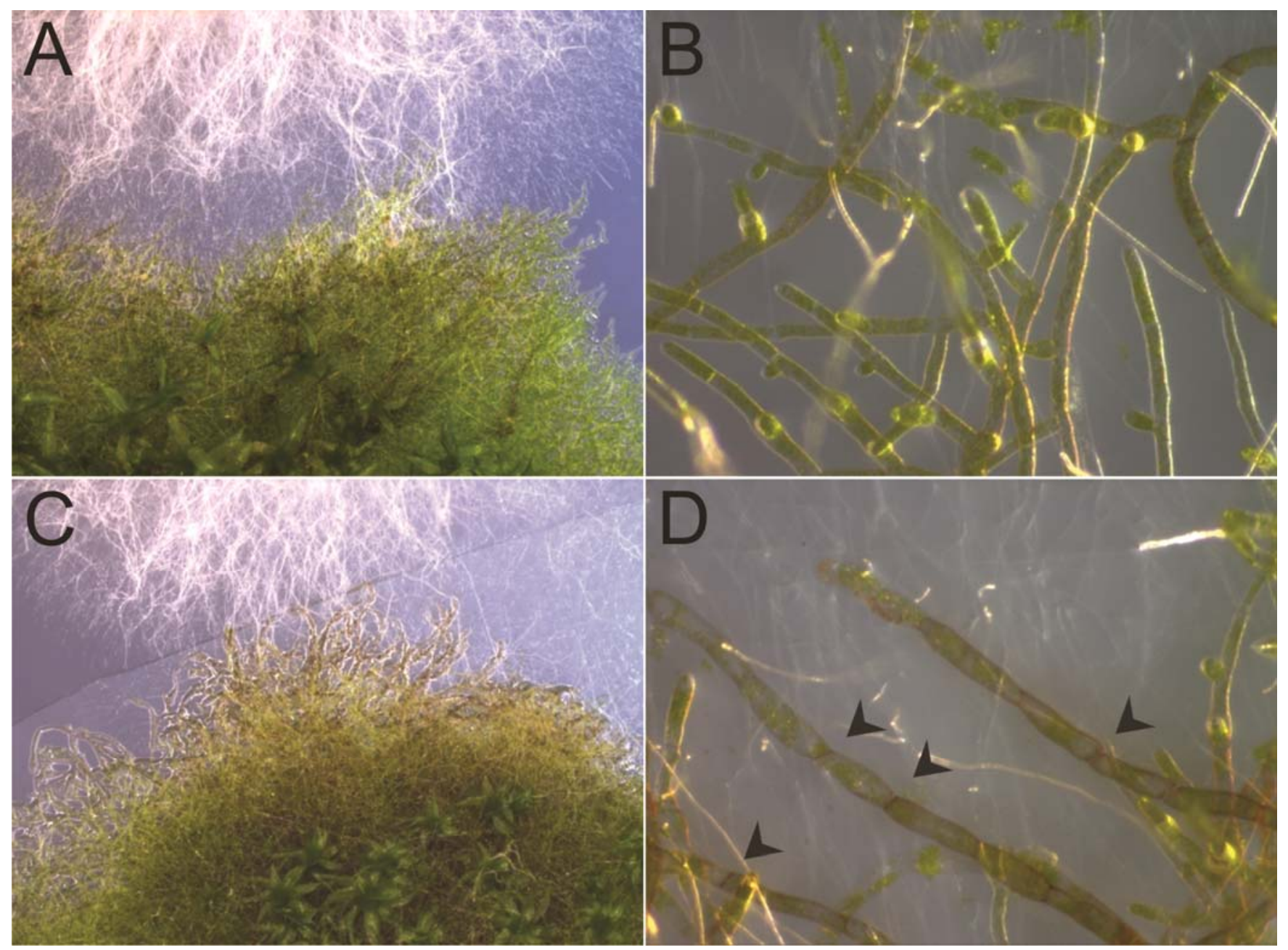

Fig. 5. Phenotypic responses of $\mathbf{A}$ and $\mathbf{B}$, wild-type Physcomitrella patens and $\mathbf{C}$ and $\mathbf{D}$, the $\triangle P$ PTSPO1 mutant to a pathogenic strain of Fusarium avenaceum at the time of initial contact with the hyphae. Inoculum was placed next to the moss colony and the fungus allowed to grow in contact with the moss protonema tissue. Arrowheads in D point out transversal cell walls that are discolored in the $\triangle P$ PTSPO1 mutant, in contrast to the wild-type moss in B. 


\section{DISCUSSION}

Results of this study showed that treatment with $\mathrm{CHN}$ caused an oxidative burst in WT $P$. patens, whereas no such response was observed in the $\Delta \operatorname{Prx} 34$ knockout mutants. Release of the peroxidase Prx34 (Lehtonen et al. 2009) and the oxidative burst occurred within seconds after treatment of $P$. patens with $\mathrm{CHN}$. These results indicate that production of superoxide, which was analyzed specifically using a quantitative chemiluminescence-based method (Tarpey et al. 2004; Uehara et al. 1993), involves Prx34 upon recognition of chitin. Prx34 is a class III peroxidase, for which two identical genes exist in the genome of $P$. patens. Both copies of the gene have been deleted in the $\Delta$ Prx34 knockout mutants, which makes them susceptible to fungal pathogens and saprophytes (Lehtonen et al. 2009). Previous studies have shown that Prx34 is pivotal to antifungal defense in $P$. patens (Lehtonen et al. 2009), and the present study revealed a plausible mechanism by which Prx34 confers resistance to fungal invaders in $P$. patens. Furthermore, the results revealed that PpTSPO1 is required for maintenance of redox homeostasis during the oxidative burst elicited by $\mathrm{CHN}$ because the $\triangle P$ PTSPO1 knockout mutant plants released more peroxidase activity to culture medium and created a stronger oxidative burst upon $\mathrm{CHN}$ treatment than was observed with WT P. patens. Previous studies (Frank et al. 2007; Vanhee et al. 2011) have not addressed the role of TSPO in the context of biotic stress. Thus, our results point out a novel host factor that is important to the maintenance of homeostasis in plants which are attacked by pathogens.

$P$. patens contains 45 putative genes for class III peroxidases but, remarkably, only $\operatorname{Prx} 34$, encoding a 35-kDa $(330$ amino acids) peroxidase protein, is responsive to $\mathrm{CHN}$ treat-
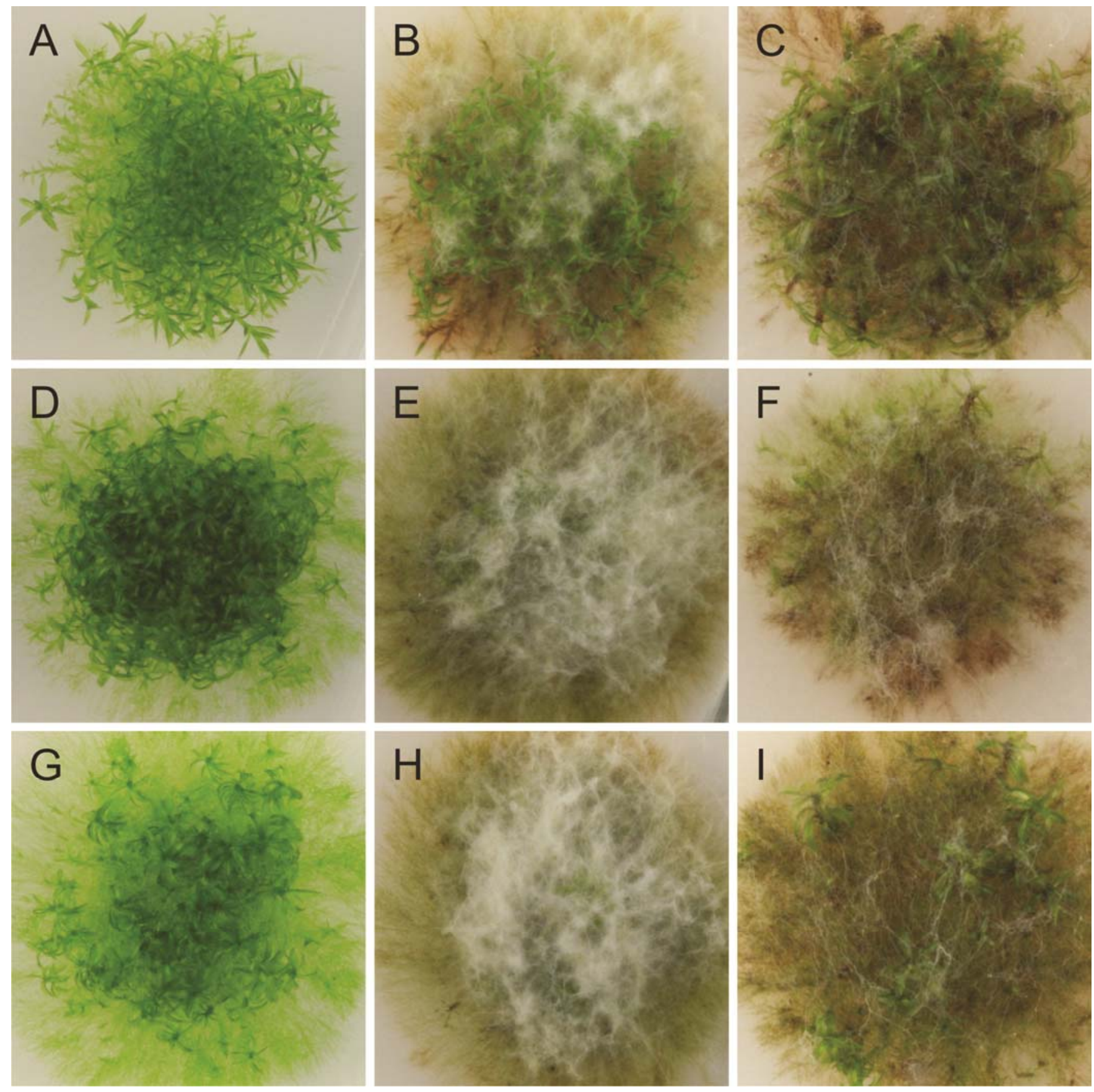

Fig. 6. Phenotypic response of $\mathbf{A}$ to $\mathbf{C}$, wild-type Physcomitrella patens and $\mathbf{D}$ to $\mathbf{F}$ and $\mathbf{G}$ to $\mathbf{I}$, two $\triangle P p T S P O 1$ mutant lines to $\mathbf{B}, \mathbf{E}$, and $\mathbf{H}$, a saprotrophic fungus (Irpex sp.) and $\mathbf{C}, \mathbf{F}$, and $\mathbf{I}$, a pathogenic fungal isolate (Fusarium avenaceum) at 12 days postinoculation. A, D, and $\mathbf{G}$, Untreated controls. 
ment (Lehtonen et al. 2009). This gene belongs to a $P$. patensspecific Prx subfamily, as indicated by phylogenetic comparison of the putative class III peroxidase genes predictable in the genome sequences of $P$. patens, $A$. thaliana, and Oryza sativa (rice) (Lehtonen et al. 2009). In vascular plants, class III peroxidases are part of the strategically positioned reservoirs of antimicrobial compounds which prevent pathogens from colonizing the tissue. They are stored in vacuoles or cell walls to be ready for excretion or release upon pathogen recognition (Almagro et al. 2009). Our results suggest that Prx34 is stored in cell walls because no oxidative burst was detected in protoplasts, in contrast to the gametophyte tissue used as a source of them. In another type of nonvascular plant, the liverwort Dumortiera hirsuta (Marchantiaphyta), a cell wall peroxidase is attributed to the production of apoplastic superoxide under drought stress (Beckett et al. 2004; Li et al. 2010). Furthermore, as we observed that treatment with $\mathrm{CHN}$ also elicits ROS production in $S$. capillifolium, a moss unrelated to $P$. patens, the data collectively suggest that a quick apoplastic oxidative burst is a common stress response in nonvascular plants.

The oxidative stress-related genes, such as $A O X 1$, respond to increased endogenous or exogenous concentrations of ROS, which can be used as an indicator for a variety of stresses (Maxwell et al. 1999; Van Aken et al. 2009). We tested by quantitative (q)PCR whether PpAOXI is induced in WT $P$. patens upon treatment with $\mathrm{CHN}$ and results were positive, which seems to be a novel observation in nonvascular plants. LOX gene activation is observed under abiotic and biotic stresses such as salt stress (Molina et al. 2002; Rodriguez-Rosales et al. 1999) and fungal infection (Hano et al. 2008). Previous studies have reported induction of $P p L O X 1$, a gene for an arachidonic acid lipoxygenase, in $P$. patens infected with fungi (Oliver et al. 2009; Ponce de Leon et al. 2007) and exhibiting intracellular accumulation of ROS revealed by nonquantitative histological staining (Olivier et al. 2009). In this study, CHN treatment was observed to induce $P p L O X 7$ that encodes a type-2 lipoxygenase located in plastids and preferring $\alpha$-linolenic acid as a substrate to produce 13(S)-hydroperoxides (Anterola et al. 2009). Furthermore, we observed induction of the gene coding for PpNOX that is homologous to the mammalian gp $91^{\text {phox }}$ respiratory burst oxidase (Torres 2010). NOX localizes in the plasma membrane and is needed for ROS accumulation and signaling in biotic and abiotic stresses (Miller et al. 2009; Torres et al. 2002). These data widened the knowledge of molecular responses to pathogen elicitors in nonvascular plants.

The $\triangle P$ PSPO1 knockout mutants were slightly but detectably and consistently more susceptible to fungal infection than WT $P$. patens in four independent experiments. In fact, the opposite was expected because the $\triangle P$ PSPOI plants produced significantly higher amounts of peroxidase activity and extracellular superoxide than WT $P$. patens. Explanations were sought from the physiological alterations described for the $\triangle$ PpTSPO1 mutants (Frank et al. 2007). These mutants are sensitive to abiotic stress and, under high salt concentration, suffer from enhanced lipid peroxidation and cell death. Our results indicated that the expression levels of the oxidative stress-related gene homologs, such as $P p A O X 1$ and $P p L O X 7$, were constitutively higher in $\triangle P$ PTSPO1 mutants than WT $P$. patens under standard growth conditions. Furthermore, the TSPO knockout mutants of Arabidopsis are also unusually sensitive to salt stress, and the higher induction of salt-responsive genes than in WT Arabidopsis indicates disturbed homeostasis (BalsemãoPires et al. 2011). Hence, the evidence indicates that the TSPOdeficient plants are under permanent oxidative stress and suffer from disturbed redox homeostasis.

Recent data of Vanhee and associates (2011) shed light on the mechanism by which the TSPO of Arabidopsis regulates redox homeostasis. Regulation is based on scavenging unbound heme and porphyrins that produce radicals under exposure to light and, consequently, damage the cellular organelles and structures (Vanhee et al. 2011). Consistent with the findings of Vanhee and associates (2011), our study shows that expression of PpTSPO1 is induced in WT P. patens upon CHN treatment (i.e., as a response to ROS accumulation), and the levels of oxidative stress-related genes are constitutively higher in the PpTSPO1 knockout mutants (i.e., in absence of the TSPO-mediated heme and porphyrin scavenging mechanism). The scavenging mechanism in Arabidopsis requires that TSPO binds heme and, also, the autophagy-related protein Atg8 via a specific Atg8 family interacting motif. The complex is subsequently degraded by autophagy (Vanhee et al. 2011). A similar role for PpTSPO1 in $P$. patens is conceivable because PpTSPO1 is implicated in translocation of protoporphyrin precursors to mitochondria for heme formation (Frank et al. 2007) and, according to our observation, the PpTSPO1 amino acid sequence (National Center for Biotechnology Information database accession number ABG37902) contains five putative Atg8 binding sites (W/Y-X-X-L/V/I) (Noda et al. 2010). Further studies are needed to show which site is active in Atg8 binding but, collectively, the findings suggest a plausible mechanism by which PpTSPO1 may suppress oxidative stress and regulate homeostasis in $P$. patens.

Higher susceptibility of the $\triangle P P T S P O 1$ knockout mutants to the saprotrophic and necrotrophic fungi tested in this study was observed as enhanced discoloration and necrotization of the protonema and gametophore tissue following contact with the fungal hyphae. The response may be associated with autophagy, whose "pro-death" functions provide an essential mechanism for removal of damaged membranes, organelles, oxidized proteins, and other cellular "garbage" and are vital for maintenance of homeostasis (Hofius et al. 2011). Autophagy can also serve "anti-death" functions, which are important in innate immunity because they limit the spread of cell death during infection with necrotophic fungal pathogens (Lenz et al. 2011). However, when the innate immunity and autophagy are insufficient to control infection and remove garbage, respectively, accumulation of host-cell-derived damage-associated molecular patterns (DAMPs) may trigger accumulation of salicylic acid and pathogenesis-related proteins (Krol et al. 2010). The DAMPs per se can be harmful, and the responses induced by them may enhance stress and cause damage to membranes (Hofius et al. 2011). These consequences from DAMP accumulation should be challenging to control in the $\triangle P$ PSPPO1 mutants that are intrinsically stressed and limited in their abilities to control ROS and homeostasis. Indeed, treatment of the $\triangle P$ PTSPOI plants with the fungal elicitor $\mathrm{CHN}$ enhanced ROS production. The salicylic-acid-mediated signaling for resistance to necrotrophic pathogens (Andersson et al. 2005) and induction of pathogenesis-related proteins by fungal elicitors (Lehtonen et al. 2009; Oliver et al. 2009; Ponce de León 2007; this study) have been reported in $P$. patens. It seems that, in the $\triangle P$ PTSPO1 mutants, additional ROS produced upon pathogen recognition may cause damage to the membranes and organelles and result in severe damage and cell death, which is advantageous to the necrotrophic fungi (Govrin and Levine 2000).

Taken together, our results point out TSPO as a novel host factor that is needed to maintain homeostasis in plants attacked by pathogens. TSPO seems to ensure that cells will retain their ability to operate the broad spectrum of defense responses against the invader despite the potentially very harmful burst of radical oxygen species as the first defensive response. Furthermore, enhanced susceptibility of plants to pathogen infections under drought and salt stress has been documented (Audenaert et al. 2002; Brosché et al. 2010; DiLeo et al. 2010). Future 
studies on TSPO, which appears as a new link in abiotic and biotic stress signaling, may help to resolve this phenomenon.

\section{MATERIALS AND METHODS}

\section{Plant material.}

Protonemal tissue of $P$. patens, ecotype Gransden (Ashton and Cove 1977), was grown in petri dishes ( $9 \mathrm{~cm}$ in diameter) on a cellophane membrane placed on BCD medium (1 mM $\mathrm{MgSO}_{4}, 1.85 \mathrm{mM} \mathrm{KH} \mathrm{PO}_{4}[\mathrm{pH} 6.5$, adjusted with $\mathrm{KOH}], 10$ $\mathrm{mM} \mathrm{KNO}{ }_{3}, 45 \mu \mathrm{M} \mathrm{FeSO}_{4}, 0.22 \mu \mathrm{M} \mathrm{CuSO}_{4}, 0.19 \mu \mathrm{M} \mathrm{ZnSO}_{4}$, $10 \mu \mathrm{M} \mathrm{H} \mathrm{BO}_{4}, 0.10 \mu \mathrm{M} \mathrm{Na} 2 \mathrm{MoO}_{4}, 2 \mu \mathrm{M} \mathrm{MnCl}, 0.23 \mu \mathrm{M}$ $\mathrm{CoCl}_{2}$, and $0.17 \mu \mathrm{M} \mathrm{KI}$ ) (Ashton and Cove 1977) supplemented with $1 \mathrm{mM} \mathrm{CaCl}, 45 \mu \mathrm{M}$ ethylenediaminetetraacetic acid disodium salt $\left(\mathrm{Na}_{2}\right.$-EDTA), and $5 \mathrm{mM}$ ammonium tartrate ([NH4 $]_{2} \mathrm{C}_{4} \mathrm{H}_{4} \mathrm{O}_{6}$ ), and solidified with $0.8 \%$ agar. The cultures were grown in a growth chamber (Model 3755; Forma Scientific, Marietta, OH, U.S.A.) at $23^{\circ} \mathrm{C}$ (photoperiod, $12 \mathrm{~h}$; light intensity, $60 \mu \mathrm{mol} \mathrm{m} \mathrm{m}^{-2} \mathrm{~s}^{-1}$ ) and subcultured weekly.

Generation of the $\triangle P$ PTSPOI and $\triangle P r x 34$ gene knockout mutants by a gene-replacement technique based on homologous recombination has been described previously (Frank et al. 2007; Lehtonen et al. 2009). Three independent $\Delta$ Prx34 mutants and both $\triangle P$ PSSPO1 mutants, all shown to have only a single integration of the knockout construct at the homologous locus and no additional insertions (Frank et al. 2007; Lehtonen et al. 2009), were included in this study.

\section{CHN treatment.}

According to the manufacturer, the product called chitosan used in this study contains chitin deacetylated to $90 \%\left(\mathrm{M}_{\mathrm{w}}<\right.$ 5000) (Kraeber GmbH \& Co, Ellerbek, Germany). It was dissolved in Milli-Q water at $10 \mathrm{mg} \mathrm{ml} \mathrm{m}^{-1}$, prefiltered twice through a 1.6- $\mu \mathrm{m}$ glass fiber filter (VWR International, West Chester, PA, U.S.A.), filter-sterilized using a $0.2-\mu \mathrm{m}$ cellulose acetate filter (Whatman $\mathrm{GmbH}$, Dassel, Germany), and stored at $-20^{\circ} \mathrm{C}$ until use. Stock solution of CHN $\left(10 \mathrm{mg} \mathrm{ml}^{-1}\right)$ was added to 1 -week-old moss liquid cultures $(2 \mathrm{ml})$ to the final concentration needed in the experiment and the moss grown as above. Sterile Milli-Q water was used as a control treatment.

\section{Superoxide production assay.}

Superoxide production in liquid moss culture medium was measured by chemiluminescence of a luciferin analog 2-methyl6-(p-methoxyphenyl)-3,7-dihydroimidazo(1,2- $\alpha$ )pyrazin-3-one (MCLA) (Sigma-Aldrich, St. Louis). Pieces of 2-week-old protonemal mats (approximately $5 \mathrm{~mm}$ in diameter) covering the LIA-plate (Lumitrac 200; Greiner Bio-One, Frickenhausen, Germany) were placed in the wells containing $5 \mu \mathrm{M}$ MCLA (final concentration) in liquid culture medium (see above). The plate was incubated in the dark at $22^{\circ} \mathrm{C}$ for 30 min before starting the measurements. Chemiluminescence was measured before addition of $\mathrm{CHN}$ and at intervals of 25 to $1,200 \mathrm{~s}$ after addition of $\mathrm{CHN}$. The measurement lasted for $1 \mathrm{~s}$ at each time point and was carried out with a VICTOR ${ }^{3}$ multilabel reader (Perkin-Elmer, Waltham, MA, U.S.A.). Differences in superoxide production in WT and knockout moss lines were calculated based on the area under the curve of measurement values in function of time (Fig. 1) and were tested by the analysis of variance (ANOVA) followed by Tukey's test $(P<0.05)$ (SPSS, v. 15.0; SPSS Inc., Chicago).

\section{Peroxidase activity assay.}

The plants for peroxidase activity assay were grown for 1 week on cellophane on the culture medium, transferred onto medium without ammonium tartrate for 1 week, and finally grown in liquid medium containing ammonium tartrate and $1 \%$ glucose for 1 week. Peroxidase activity was tested in aliquots $(50 \mu \mathrm{l})$ of the liquid culture medium spun at $16,000 \times g$ for 1 min. The supernatant was transferred to a clean tube and kept on ice until the measurement. The reaction mixture $(1 \mathrm{ml})$ contained $0.5 \mathrm{mM}$ 2,2'-azino-bis3-ethylbenzthiazoline-6-sulphonic acid (ABTS) (Fluka, Buchs, Switzerland) and $200 \mu \mathrm{M} \mathrm{H}_{2} \mathrm{O}_{2}$ in phosphate-citrate buffer $(29 \mathrm{mM}$ phosphate and $23 \mathrm{mM}$ citrate, $\mathrm{pH}$ 4.0) preheated to $35^{\circ} \mathrm{C}$ in a heated cuvette chamber before adding the sample $(40 \mu \mathrm{l})$. The absorbance at $405 \mathrm{~nm}\left(\mathrm{~A}_{405}\right)$ was recorded using a spectrophotometer (UV-1700; Shimazu, Kyoto, Japan) 15 to $120 \mathrm{~s}$ after starting the reaction. Peroxidase activity was calculated based on oxidation of ABTS as moles per liter per second per gram of fresh moss tissue. The $\mathrm{A}_{405}$ extinction coefficient of ABTS is $36,800 \mathrm{M}^{-1} \mathrm{~cm}^{-1}$. Three replicates (cultures) of the moss treated or untreated with $\mathrm{CHN}$ were included in each of the three independent experiments.

\section{Fungal isolates and inoculation.}

A saprophytic fungal isolate of an Irpex sp. (Basidiomycota; Polyporales; family Steccherinaceae) and a pathogenic isolate of $F$. avenaceum (Hyphomycetes) were both isolated from the Sunagoke moss (Racomitrium japonicum Dozy and Molk.; family Grimmiaceae) growing in the field in Japan and have been described previously (Akita et al. 2011; Lehtonen et al. 2009). Pure cultures of these fungi were grown on potato dextrose agar (Biokar Diagnostics, Pantin Cedex, France) and used for inoculation.

Colonies of the WT and $\triangle P p T S P O 1$ mutants of $P$. patens were grown for inoculation on cellophane on agar media as described for plant materials above. When 1 month old, the colonies were inoculated with fungi using a small plug of culture medium excised from the edge of an actively growing fungal colony. Inoculum was placed next to the moss colony, allowing

Table 1. Primers designed for quantitative polymerase chain reaction analysis of gene expression utilizing the genome sequence of Physcomitrella patens available at the COSMOSS website

\begin{tabular}{|c|c|c|}
\hline Gene & Joint Genome Institute gene model & Sequence $5^{\prime}$ to $3^{\prime}$ \\
\hline$E F 1 \alpha$ & Phypa_158916 & $\begin{array}{l}\text { fwd AGGCAGACGTGGGTACTTGA } \\
\text { rev CATTAATCATCAGGCAGGCATA }\end{array}$ \\
\hline$L 21$ & Phypa_106900 & $\begin{array}{l}\text { fwd TGGTGTCGTGATGAACAAGC } \\
\text { rev CTCAATTCGAACGTGCAGAC }\end{array}$ \\
\hline РpTSPO1 & Phypa_148670 & $\begin{array}{l}\text { fwd CGTGGTTAGTGTGGGCTGA } \\
\text { rev AGTAAGCACCGAGAGCGAAA }\end{array}$ \\
\hline РpАох & Phypa_89421 & $\begin{array}{l}\text { fwd TCGTGACGTTAACCATTTCG } \\
\text { rev GATTCCCGCAGCTCCTTAC }\end{array}$ \\
\hline PpNox & Phypa_204103 & $\begin{array}{l}\text { fwd CACGATGTTGCAGTCGTTG } \\
\text { rev TACGTGCCCTAGTGCCTGA }\end{array}$ \\
\hline PpLOX7 & Phypa_184078 & $\begin{array}{l}\text { fwd GTGGCGGTTTGATCAGGA } \\
\text { rev CGTTCAGCCATCCCTCTTC }\end{array}$ \\
\hline $\operatorname{Prx} 34$ & Phypa_144797/144819 & $\begin{array}{l}\text { fwd CAATACGCTACTCGCGACTCTGT } \\
\text { rev CGTCTCTTCGACCGCCATA }\end{array}$ \\
\hline
\end{tabular}


the hyphae to grow in contact with moss tissue. Inoculated moss colonies were cultured under the conditions described above.

\section{RNA extraction, cDNA synthesis, and qPCR.}

Two cultures each of the CHN-treated and untreated $\triangle P$ PSPOI mutant and WT P. patens were grown in each experiment and used for extraction of total RNA. Moss tissue was ground to powder under liquid nitrogen. Total RNA was extracted using a Trizol-like reagent (Caldo et al. 2004). Total RNA $(1 \mu \mathrm{g})$ was treated with RQ1 RNase-free DNase (Promega Corp., Fitchburg, WI, U.S.A.) according to the manufacturer's instructions.

Expression levels of the genes of interest (Table 1) were estimated using qPCR. For cDNA synthesis, $1 \mu \mathrm{g}$ of the DNase-treated RNA was incubated with $1 \mu \mathrm{l}$ (200 ng) of random hexamers (Sigma Genosys, Woodlands, TX, U.S.A.) at $70^{\circ} \mathrm{C}$ for $10 \mathrm{~min}$ and added to the cDNA reaction mixture consisting of $4 \mu \mathrm{l}$ of $5 \times$ Moloney murine leukaemia virus (MMLV) reverse-transcription (RT) buffer, $2 \mu \mathrm{l}$ of $0.1 \mathrm{M}$ dithiotreitol, $1 \mu \mathrm{l}$ of $10 \mathrm{mM}$ dNTPs, $0.5 \mu \mathrm{l}(20 \mathrm{U})$ of RNasin (Promega Corp.), and $2 \mu \mathrm{l}$ (400 U) of M-MLV RT (Promega Corp.). The reaction mixture was incubated at $37^{\circ} \mathrm{C}$ for $1 \mathrm{~h}$ and, subsequently, at $70^{\circ} \mathrm{C}$ for $10 \mathrm{~min}$, after which $35 \mu \mathrm{l}$ of nucleasefree water was added.

Primers (Table 1) for the target genes were designed utilizing the genome sequence of $P$. patens (Rensing et al. 2008) available online at the COSMOSS website.

cDNA of the two replicate samples from each moss line and treatment were pooled for qPCR analysis. Three technical replicates of each sample $(2.5 \mu \mathrm{l}$ of cDNA in a total volume of 10 $\mu \mathrm{l}$ of reaction mix) were included. qPCR was carried out using LightCycler 480 SYBR Green I Master mix (Roche, Basel, Switzerland) and the LightCycler 480 SYBR Green system following the manufacturer's instructions. EFl $\alpha$ and $L 21$ (Table 1) were used as reference genes to normalize possible variation in the amounts of the cDNA template between samples. Target specificity of the primers was verified by sequencing the amplification products. Gene expression levels were calculated employing relative quantification with efficiency correction (Pfaffl 2001).

\section{Statistical analysis.}

ANOVA followed by Tukey's test for significant differences was used to analyze the data on ROS and peroxidase production and the fold values from qPCR analyses. The fold values were $\log _{2}$-transformed and tested by the between the $\mathrm{CHN}$ treated and control samples $(P<0.05)$ (SPSS, v. 15.0, SPSS Inc.). The differences in gene transcript levels between WT $P$. patens and $\triangle P$ PTSPO1 mutant were tested by a paired-sample $t$ test using the same program $(P<0.05)$.

\section{ACKNOWLEDGMENTS}

This study was supported by the Academy of Finland (grants 111866, 1134759 and 1253126), the Finnish Graduate School of Plant Biology, and the Excellence Initiative of the German Federal and State Governments (EXC 294).

\section{LITERATURE CITED}

Akita, M., Lehtonen, M. T., Koponen, H., Marttinen, E. M., and Valkonen, J. P. T. 2011. Infection of the Sunagoke moss panels with fungal pathogens hampers sustainable greening in urban environments. Sci. Total. Environ. 409:3166-3173.

Almagro, L., Gómez Ros, L. V., Belchi-Navarro, S., Bru, R., Ros Barceló, A., and Pedreño, M. A. 2009. Class III peroxidases in plant defence reactions. J. Exp. Bot. 60:377-390.

Andersson, R. A., Akita, M., Pirhonen, M., Gammelgård, E., and Valkonen, J. P. T. 2005. Moss-Erwinia pathosystem reveals possible similarities in pathogenesis and pathogen defense in vascular and nonvascular plants. J. Gen. Plant. Pathol. 71:23-28.

Angelova, M. B., Pashova, S. B., Spasova, B. K., Vassilev, S. V., and Slokoska, L. S. 2005. Oxidative stress response of filamentous fungi induced by hydrogen peroxide and paraquat. Mycol. Res. 109:150-158.

Anterola, A., Göbel, C., Hornung, E., Sellhorn, G., Feussner, I., and Grimes, H. 2009. Physcomitrella patens has lipoxygenases for both eicosanoid and octadecanoid pathways. Phytochemistry 70:40-52.

Apel, K., and Hirt, H. 2004. Reactive oxygen species: Metabolism, oxidative stress, and signal transduction. Annu. Rev. Plant Biol. 55:373-399.

Ashton, N. W., and Cove, D. J. 1977. The isolation and preliminary characterisation of auxotrophic and analogue resistant mutants of the moss, Physcomitrella patens. Mol. Gen. Genet. 154:87-95.

Audenaert, K., De Meyer, G. B., and Hofte, M. M. 2002. Abscisic acid determines basal susceptibility of tomato to Botrytis cinerea and suppresses salicylic acid-dependent signaling mechanisms. Plant Physiol. 128:491-501

Balsemão-Pires, E., Jaillais, Y., Olson, B., Andrade, L., Umen, J., Chory, J., and Sachetto-Martins, G. 2011. The Arabidopsis translocator protein (AtTSPO) is regulated at multiple levels in response to salt stress and perturbations in tetrapyrrole metabolism. BMC Plant Biol. 11:108.

Beckett, R., Minibayeva, F., Luthje, S., and Bottger, M. 2004. Reactive oxygen species metabolism in desiccation-stressed thalli of the liverwort Dumortiera hirsuta. Physiol. Plant. 122:3-10.

Bindschedler, L., Dewdney, J., Blee, K., Stone, J., Asai, T., Plotnikov, J., Denoux, C., Hayes, T., Gerrish, C., Davies, D., Ausubel, F., and Bolwell, G. 2006. Peroxidase-dependent apoplastic oxidative burst in Arabidopsis required for pathogen resistance. Plant J. 47:851-863.

Brosché, M., Overmyer, K., Wrzaczek, M., Kangasjärvi, J., and Kangasjärvi, S. 2010. Stress signaling III: Reactive oxygen species. Pages 91-102 in: Abiotic Stress Adaptation in Plants: Physiological, Molecular and Genomic Foundation. A. Pareek, S. K. Sopory, H. J. Bohnert, and Govindjee, eds. Springer, Dordrecht, The Netherlands.

Caldo, R. A., Nettleton, D., and Wise, R. P. 2004. Interaction-dependent gene expression in Mla-specified response to barley powdery mildew. Plant Cell 16:2514-2528.

Cove, D. 2005. The moss Physcomitrella patens. Annu. Rev. Genet. 39:339-358.

Cuming, A. C. 2009. Plant-pathogen interactions: A view from the evolutionary basement. New Phytol. 183:237-239.

DiLeo, M. V., Pye, M. F., Roubtsova, T. V., Duniway, J. M., MacDonald, J. D., Rizzo, D. M., and Bostock, R. M. 2010. Abscisic acid in salt stress predisposition to Phytophthora root and crown rot in tomato and chrysanthemum. Phytopathology 100:871-879.

Frank, W., Baar, K., Qudeimat, E., Woriedh, M., Alawady, A., Ratnadewi, D., Gremillon, L., Grimm, B., and Reski, R. 2007. A mitochondrial protein homologous to the mammalian peripheral-type benzodiazepine receptor is essential for stress adaptation in plants. Plant $\mathrm{J}$. 51:1004-1018.

Fujita, M., Fujita, Y., Noutoshi, Y., Takahashi, F., Narusaka, Y., YamaguchiShinozaki, K., and Shinozaki, K. 2006. Crosstalk between abiotic and biotic stress responses: A current view from the points of convergence in the stress signaling networks. Curr. Opin. Plant Biol. 9:436-442.

Govrin, E. M., and Levine, A. 2000. The hypersensitive response facilitates plant infection by the necrotrophic pathogen Botrytis cinerea. Curr. Biol. 10:751-757.

Hano, C., Addi, M., Fliniaux, O., Bensaddek, L., Duverger, E., Mesnard, F., Lamblin, F., and Laine, E. 2008. Molecular characterization of cell death induced by a compatible interaction between Fusarium oxysporum f. sp. linii and flax (Linum usitatissimum) cells. Plant Physiol. Biochem. 46:590-600.

Hofius, D., Munch, D., Bressendorff, S., Mundy, J., and Petersen, M 2011. Role of autophagy in disease resistance and hypersensitive response-associated cell death. Cell Death Differ. 18:1257-1262.

Kawano, T. 2003. Roles of the reactive oxygen species-generating peroxidase reactions in plant defense and growth induction. Plant Cell Rep. 21:829-837.

Keller, T., Damude, H., Werner, D., Doerner, P., Dixon, R., and Lamb, C. 1998. A plant homolog of the neutrophil NADPH oxidase gp91 ${ }^{\text {phox }}$ subunit gene encodes a plasma membrane protein with $\mathrm{Ca}^{2+}$ binding motifs. Plant Cell 10:255-266

Knaul, J. Z., Hudson, S. M., and Creber, K. A. M. 1999. Improved mechanical properties of chitosan fibers. J. Appl. Polym. Sci. 72:1721-1732.

Krol, E., Mentzel, T., Chinchilla, D., Boller, T., Felix, G., Kemmerling, B., Postel, S., Arents, M., Jeworutzki, E., Al-Rasheid, K. A. S., Becker, D. and Hedrich, R. 2010. Perception of the Arabidopsis danger signal peptide 1 involves the pattern recognition receptor AtPEPR1 and its close homologue AtPEPR2. J. Biol. Chem. 285:13471-13479.

Lawton, M., and Saidasan, H. 2009. Pathogenesis in mosses. Annu. Plant Rev. 36:298-339. 
Lehtonen, M. T., Akita, M., Kalkkinen, N., Ahola-Iivarinen, E., Rönnholm, G., Somervuo, P., Thelander, M., and Valkonen, J. P. T. 2009. Quicklyreleased peroxidase of moss in defense against fungal invaders. New Phytol. 183:432-443.

Lenz, H. D., Haller, E., Melzer, E., Gust, A. A., and Nuernberger, T. 2011. Autophagy controls plant basal immunity in a pathogenic lifestyledependent manner. Autophagy 7:773-774.

Li, J. L. Y., Sulaiman, M., Beckett, R. P., and Minibayeva, F. V. 2010. Cell wall peroxidases in the liverwort Dumortiera hirsuta are responsible for extracellular superoxide production, and can display tyrosinase activity. Physiol. Plant. 138:474-484.

Mayaba, N., Minibayeva, F., and Beckett, R. 2002. An oxidative burst of hydrogen peroxide during rehydration following desiccation in the moss Atrichum androgynum. New Phytol. 155:275-283.

Maxwell, D. P., Wang, Y., and McIntosh, L. 1999. The alternative oxidase lowers mitochondrial reactive oxygen production in plant cells. Proc. Natl. Acad. Sci. U.S.A. 96:8271-8276.

Miller, G., Schlauch, K., Tam, R., Cortes, D., Torres, M. A., Shulaev, V., Dangl, J. L., and Mittler R. 2009. The plant NADPH oxidase RBOHD mediates rapid systemic signaling in response to diverse stimuli. Sci. Signal. 2:ra45.

Mochizuki, N., Tanaka, R., Grimm, B., Masuda, T., Moulin, M., Smith, A. G., Tanaka, A., and Terry, M. J. 2010. The cell biology of tetrapyrroles: A life and death struggle. Trends Plant Sci. 15:488-498.

Molina, A., Bueno, P., Marín, M. C., Rodríguez-Rosales, M. P., Belver, A., Venema, K., and Donaire, J. P. 2002. Involvement of endogenous salicylic acid content, lipoxygenase and antioxidant enzyme activities in the response of tomato cell suspension cultures to $\mathrm{NaCl}$. New Phytol. 156:409-415

Nanda, A. K., Andrio, E., Marino, D., Pauly, N., and Dunand, C. 2010. Reactive oxygen species during plant-microorganism early interactions. J. Integr. Plant Biol. 52:195-204.

Oliver, J. P., Castro, A., Gaggero, C., Cascón, T., Schmelz, E. A., Castresana, C., and Ponce de León I. 2009. Pythium infection activates conserved plant defense responses in mosses. Planta 230:569-579.

Noda, N. N., Ohsumi, Y., and Inagaki, F. 2010. Atg8-family interacting motif crucial for selective autophagy. FEBS (Fed. Eur. Biochem. Soc.) Lett. 584:1379-1385.

Pfaffl, M. W. 2001. A new mathematical model for relative quantification in real-time RT-PCR. Nucleic Acids Res. 29:e45-e45.

Ponce de León, I., Oliver, J. P., Castro, A., Gaggero, C., Bentancor, M., and Vidal, S. 2007. Erwinia carotovora elicitors and Botrytis cinerea activate defense responses in Physcomitrella patens. BMC Plant Biol. 7:52.

Proctor, M. C. F., Ligrone, R., and Duckett, J. G. 2007. Desiccation tolerance in the moss Polytrichum formosum: Physiological and fine-structural changes during desiccation and recovery. Ann. Bot. 99:75-93.

Rensing, S. A., Lang, D., Zimmer, A. D., Terry, A., Salamov, A., Shapiro, H., Nishiyama, T., Perroud, P.-F., Lindquist, E., Kamisugi, Y., Tanahashi, T., Sakakibara, K., Fujita, T., Oishi, K., Shin-I, T., Kuroki, Y., Toyoda, A., Suzuki, Y, Hashimoto, S., Yamaguchi, K., Sugano, S., Kohara, Y., Fujiyama, A., Anterola, A., Aoki, S., Ashton, N., Barbazuk, W. B., Barker, E., Bennetzen, J., Blankenship, R., Cho, S. H., Dutcher, S. K., Estelle, M., Fawcett, J. A., Gundlach, H., Hanada, K., Hey, A., Hicks,
K. A., Hughes, J., Lohr, M., Mayer, K., Melkozernov, A., Murata, T., Nelson, D., Pils, B., Prigge, M., Reiss, B., Renner, T., Rombauts, S., Rushton, P., Sanderfoot, A., Schween, G., Shiu, S.-H., Stueber, K., Theodoulou, F. L., Tu, H., de Peer, Y. V., Verrier, P. J., Waters, E., Wood, A., Yang, L., Cove, D., Cuming, A. C., Hasebe, M., Lucas, S., Mishler, B. D., Reski, R., Grigoriev, I. V., Quatrano, R. S., and Boore J. L. 2008. The Physcomitrella genome reveals evolutionary insights into the conquest of land by plants. Science 319:64-69.

Rodriguez-Rosales, M., Kerkeb, L., Bueno, P., and Donaire, J. 1999. Changes induced by $\mathrm{NaCl}$ in lipid content and composition, lipoxygenase, plasma membrane $\mathrm{H}^{+}$-ATPase and antioxidant enzyme activities of tomato (Lycopersicon esculentum. Mill) calli. Plant Sci. 143:143-150.

Schuller, D., Ban, N., Vanhuystee, R., McPherson, A., and Poulos, T. 1996. The crystal structure of peanut peroxidase. Structure 4:311-321.

Shibuya, N., and Minami, E. 2001. Oligosaccharide signalling for defence responses in plant. Physiol. Mol. Plant Pathol. 59:223-233.

Strepp, R., Scholz, S., Kruse, S., Speth, V., and Reski, R. 1998. Plant nuclear gene knockout reveals a role in plastid division for the homolog of the bacterial cell division protein FtsZ, an ancestral tubulin. Proc. Natl. Acad. Sci. U.S.A. 95:4368-4373.

Tarpey, M. M., Wink, D. A., and Grisham, M. B. 2004. Methods for detection of reactive metabolites of oxygen and nitrogen: In vitro and in vivo considerations. Am. J. Physiol. Regul. Integr. Comp. Physiol. 286:R431-R444.

Torres, M. 2010. ROS in biotic interactions. Physiol. Plant. 138:414-429.

Torres, M. A., Dangl, J. L., and Jones, J. D. G. 2002. Arabidopsis gp $91^{\text {phox }}$ homologues AtrbohD and AtrbohF are required for accumulation of reactive oxygen intermediates in the plant defense response. Proc. Natl. Acad. Sci. U.S.A. 99:517-522.

Uehara, K., Maruyama, N., Huang, C.K., and Nakano, M. 1993. The first application of a chemiluminescence probe, 2-methyl-6-[p-methoxyphenyl]-3,7-dihydroimidazo[1,2-a]pyrazin-3-one (MCLA), for detecting $\mathrm{O}_{2}$-production, in vitro, from Kupffer cells stimulated by phorbol myristate acetate. FEBS (Fed. Eur. Biochem. Soc.) Lett. 335:167-170.

Van Aken, O., Zhang, B., Carrie, C., Uggalla, V., Paynter, E., Giraud, E., and Whelan, J. 2009. Defining the Mitochondrial Stress Response in Arabidopsis thaliana. Mol. Plant 2:1310-1324.

Vanhee, C., Zapotoczny, G., Masquelier, D., Ghislain, M., and Batoko, H. 2011. The Arabidopsis multistress regulator TSPO is a heme binding membrane protein and a potential scavenger of porphyrins via an autophagy-dependent degradation mechanism. Plant Cell 23:785 -805.

Walley, J. W., Coughlan, S., Hudson, M. E., Covington, M. F., Kaspi, R., Banu, G., Harmer, S. L., and Dehesh, K. 2007. Mechanical stress induces biotic and abiotic stress responses via a novel cis-element. PLoS Genet. 3:e172.

Wojtaszek, P. 1997. Oxidative burst: An early plant response to pathogen infection. Biochem. J. 322:681-692.

\section{AUTHOR-RECOMMENDED INTERNET RESOURCE}

The COSMOSS moss computational biology toolbox: www.cosmoss.org 